\title{
The lived experience of healthy adults following a ketogenic diet:
} A qualitative study

\author{
Journal of Holistic Performance | ISSN: 2463-7238 | Published: 4 May 2018 \\ Cliff J. d C. Harvey, ${ }^{1}$ Grant M. Schofield, ${ }^{1}$ Micalla Williden ${ }^{1}$ \\ cliff@hpn.ac.nz
}

\begin{abstract}
Background. Very-low-carbohydrate ketogenic diets (VLCKDs) promote benefits for a range of health conditions. However, there is little research elucidating the 'lived experience' of individuals undertaking these diets, and the effects of keto-induction, during adaptation to these diets.

Objective. This study aimed to evaluate the subjective experiences of people following a very low carbohydrate, ketogenic diet.

Design. This paper describes the qualitative experiences of twenty-eight non-obese, non-diabetic participants, (2 males, 26 females: age \pm SD: $35 \pm 4$ y) in a randomised controlled trial to test the effects of medium chain triglyceride (MCT) supplementation in a VLCKD on time to nutritional ketosis (NK), symptoms of keto-induction, and mood. The experience provided by the diet was rated by a free-form daily diary entry and post-study focus group. Diary entries and focus group transcription were coded inductively and grouped into common themes.
\end{abstract}

Results. Twenty-three participants completed the 3-week study. Physical effects accounted for over $28 \%$ of references. Other results were categorised as; mood, energy and cognition (23\%), satiety and hunger (16\%), cravings and temptation (11\%), and sleep (8\%). Overall, $49 \%$ of references were classified as 'positive' with $8 \%$ neutral, and $43 \%$ negative. Positive impressions were higher after participants had achieved nutritional ketosis and negative impressions higher during keto-induction. Negative impressions, both concerning physical symptoms and feelings of mood and well-being, tended towards improvement over the course of the study, and positive impressions improved. However, there was a large variation in responses, and several respondents reported adverse effects throughout the study.

Conclusion. Despite challenges, especially gastrointestinal effects, the overall perception of the diet was positive, and it provided benefits for wellbeing, mood, sleep, and sugar cravings which tended towards improvement over the course of the study. Negative experiences decreased as participants adapted to the VLCKD. Most participants continued post-study, using a lower-carbohydrate diet, due to these perceived benefits. Our findings suggest that the experience of a VLCKD is positive but variable. Further research on individual tolerance and response to low carbohydrate diets is warranted. 


\section{Introduction.}

Low-carbohydrate, high-fat (LCHF) and very low-carbohydrate ketogenic diets (VLCKDs) offer specific benefits for health conditions ranging from neurological disorders, cancer, obesity, diabetes and other conditions on the spectrum of metabolic syndrome. ${ }^{1-11}$ Adaptation to a ketogenic diet (keto-adaptation) facilitates the improved use of lipids for fuel and offers the potential for cognitive and physical performance enhancement. Thus, they are becoming increasingly popular for mainstream and athletic use for a range of outcomes including weight-loss and maintenance, ${ }^{12}$ improved satiety and a reduction in hunger. ${ }^{13-15}$ Ketogenic diets result in 'nutritional ketosis' (NK) that is distinct from pathological ketoses, such as diabetic ketoacidosis (DKA), ${ }^{16,} 17$ the condition characterised by a triad of hyperglycaemia (blood glucose $>11 \mathrm{mmol} . \mathrm{L}^{-1}$ ), increased total body ketone concentration of $>$ 3 mmol.L $\mathrm{L}^{-1},{ }^{18}$ and resultant metabolic acidosis. ${ }^{17}$ VLCKDs typically result in betahydroxybutyrate (BOHB) levels of $\geq 0.5$ mmol.L $\mathrm{L}^{-1} \quad 19$ and $<5$ mmol.L $\mathrm{L}^{-1}$ without hyperglycaemia, and this level has been used previously as indicative of entry into NK. ${ }^{20}$

Adaptation from a standard, highercarbohydrate diet, to a VLCKD, and the induction of ketosis (keto-induction) can cause various unpleasant symptoms, for several days. ${ }^{21}$ These symptoms can be referred to, in common parlance, as 'keto-flu' but are not well elucidated in the scientific literature. For example, a Google search returns over 22,000 results for the term "keto-flu" but the same term searched in MEDLINE Complete, CINAHL Complete, Alt HealthWatch, Food Science Source, SPORTDiscuss with Full Text, Psychology, and the EBSCO Behavioural
Sciences Collection returns no results. Symptoms of keto-induction are predominantly constipation, headache, halitosis, muscle cramps, diarrhoea, and general weakness and rash. ${ }^{22}$ These symptoms result from increased natriuresis, kaliuresis, and diuresis in response to lowered insulin levels, transient reductions in glucose provision to the brain, and constipation resulting from reduced food volume or reduced fibre intake.23-26 These factors and resultant adverse effects are typically limited to the first 1-4 days of a ketogenic diet. ${ }^{23,} 27$

Yancy and colleagues noted an 8\% drop out rate due to difficulties adhering to an LCHF diet, with a further $5 \%$ withdrawing from their study due to adverse effects. ${ }^{22}$ High attrition rates due to tolerability and gastrointestinal side effects also occur in childhood epilepsy research. ${ }^{3}$ However, few studies have specifically looked at the human experience of a ketogenic diet and symptoms of keto-induction, keto-adaptation and personal reflections on the challenges and opportunities of the diet. Studies have noted common adverse physical effects, such as dehydration and gastrointestinal (GI) disturbances. ${ }^{28,} 29$ However, these studies did not investigate the qualitative, 'lived experience' of participants undertaking a VLCKD. The experience of any dietary intervention is likely to affect compliance and adherence, and in the absence of significant differences between outcomes from different diets, an improved human experience while following a diet, could provide for relative superiority due to ease, comfort and enjoyment.

The overarching aim of this study was to evaluate broad themes of experience within a ketogenic diet and to provide 'snapshots' of individual experiences of a ketogenic diet. This 
paper aims to help clinicians understand better the challenges and opportunities presented by the diet and to help inform further research that may be of use to those following ketogenic diets.

\section{Methods}

\section{Study design}

This research reports on qualitative data collected within a randomised controlled trial comparing the use of medium chain triglycerides (MCT) to long-chain triglycerides in a classic (4:1 lipid to non-lipid ratio) ketogenic diet. Daily diary entries were recorded in an online questionnaire using Google Forms online software, and a post-study focus group was facilitated by one of the research team and recorded and transcribed by the primary researcher. The research was conducted in accordance with AUT ethical guidelines. Ethics approval was provided by the AUT University ethics committee, approval number 15/317. All participants were briefed twice on the intervention and study and gave written informed consent.

\section{Participants and setting}

Twenty-eight participants (2 males, 26 females: age \pm SD: $35 \pm 4$ y) (table 1) were recruited between the $18^{\text {th }}$ and $19^{\text {th }}$ of October 2015 by a 'snowball method'so from the researchers' social media networks, with sharing encouraged to facilitate a 'viral' spread of recruitment and a broad demographic range of participants. All participants gave written, informed consent to participate in a randomised, double-blinded, placebocontrolled study. Participants were required to be non-obese $(<30 \mathrm{BMI}$ ), not diagnosed as diabetic, not currently nor previously following a ketogenic diet and not a client of any of the researchers in clinical practice.

Table 1. Demographic characteristics of participants

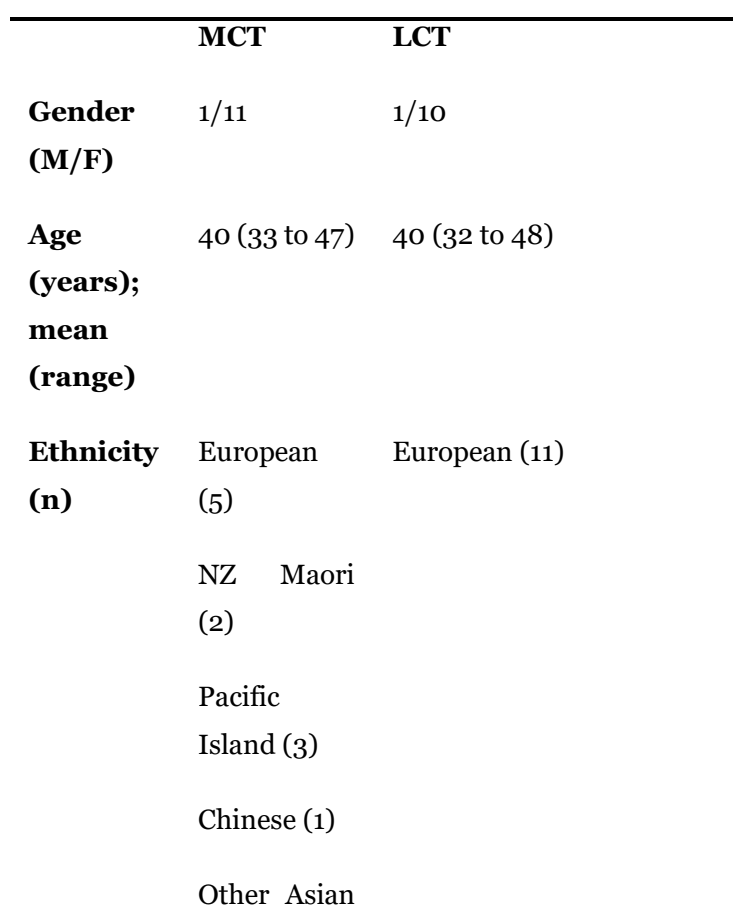

(1)

MCT: medium chain triglyceride, LCT: long chain triglyceride, M: male, F: female.

The study took place between the $2^{\text {nd }}$ and $21^{\text {st }}$ of November 2015. Collection of data and analysis was performed at AUT Human Potential Centre, Auckland, New Zealand. (Figure 1.) The trial was registered by the Australia New Zealand Clinical Trial Registry. ACTRN12616001099415. 


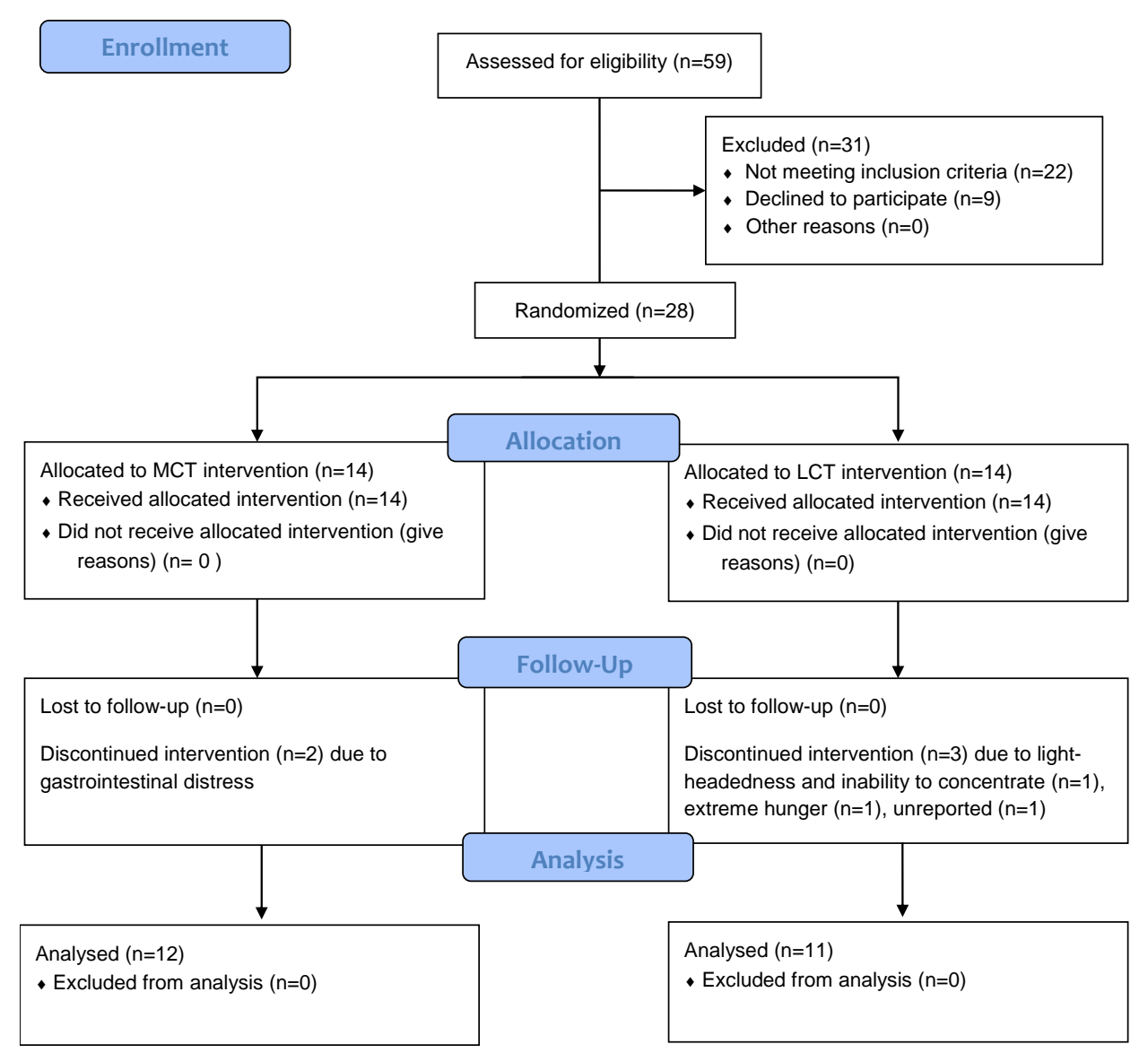

Figure 1. Participant flow diagram

Participants were prescribed a ketogenic diet plan with a 4:1 lipid to non-lipid ratio. Males were allocated a diet containing $2200 \mathrm{kcal}^{-d_{a}{ }^{-}}$ ${ }^{1}$ and females 1800 kcal.day $^{-1}$ each equating to $80 \%$ calories from fat (including supplemental oils), 13 to $17 \%$ from protein and 3 to $6 \%$ from carbohydrate. Minor differences in carbohydrate and protein were due to the use of protein intake of $1.4 \mathrm{~g}^{\mathrm{kg}} \mathrm{kg}^{-1}$ (population means for male and female respectively) bm.day-1 ${ }^{-1}$ consistent with International Society of Sports Nutrition (ISSN) guidelines for optimal protein intake for performance. ${ }^{31}$ Participants were randomised to receive either an MCT supplement containing $65 \%$ caprylic acid (C:8) and $35 \%$ capric acid (Amtrade NZ limited) as triglyceride or a long chain triglyceride (sunflower) oil (Home Brand), $30 \mathrm{ml}$, three times per day, for 20 days. Additionally, participants were provided with two introductory workshops to elucidate the dietary plan and its use. This paper reports the overall, qualitative impressions of a ketogenic diet across the intervention. Diary entries and focus group transcription were coded inductively and grouped into common themes. Twenty-three participants completed the study. Two withdrew from the MCT group (illness and gastrointestinal discomfort) and three (one due to extreme hunger, one unreported and a third due to lightheadedness and inability to concentrate) from the LCT group. These results were omitted from this analysis. Participants were instructed to contact the primary and 
tertiary researchers for any assistance during the study duration. $\mathrm{CH}$ is a registered clinical nutritionist with the New Zealand Clinical Nutrition Association, and MW is a registered nutritionist with the Nutrition Society of New Zealand.

\section{Qualitative measures}

Participants entered daily diary entries into an online questionnaire in addition to the measures taken for the RCT above, namely: blood glucose, beta-hydroxybutyrate, Profile of Mood States (POMS), and a ketogenic diet symptom questionnaire developed by the primary researcher (C.H.) based on commonly reported symptoms resulting from ketoinduction. These quantitative measures will be reported in a separate paper.

The questionnaire asked the participants to respond to the open-ended, free-form question: "Please tell us about the experience of this diet. Describe in your own words anything that you may have felt or experienced (in the last 24 hours) because of this process". Additionally, an informal, semi-structured, post-study focus group was conducted. Questions posed in the focus group were also open-ended to facilitate ongoing discussion, e.g. "How did you feel while following the diet?", "How did the diet differ from your normal diet?", "Why did you enjoy, or not enjoy, the diet you were on?”, "What effects, if any, did you notice while on the diet?"

\section{Results}

Diary entries and focus-group transcriptions were coded using an inductive approach by the lead researcher, with 'triangulation' (checking and confirmation) provided by the secondary and tertiary researchers to ensure robustness and validity of the themes and codes identified. In total 458 diary entries were submitted by the participants along with the post-study focus group. 830 references were extracted from the diaries and transcription. Physical effects accounted for over $28 \%$ of references. Other results were categorised as; mood, energy and cognition (23\%), satiety and hunger (16\%), cravings and temptation (11\%), and sleep (8\%). Additionally, it was noted when participants directly stated that they were 'feeling good' and references related to behavioural change were noted to clarify the themes.

Overall, $49 \%$ of references were classified as 'positive' with $8 \%$ neutral, and $43 \%$ negative. Positive impressions were higher after participants had achieved NK and negative impressions higher during keto-induction. (Figure 2.) Adverse effects overall, both concerning physical symptoms and feelings of mood and well-being, tended to improve over the course of the study, and conversely, positive impressions improved. However, it was noted that there was a large variation in responses and several respondents reported adverse effects throughout the study. 


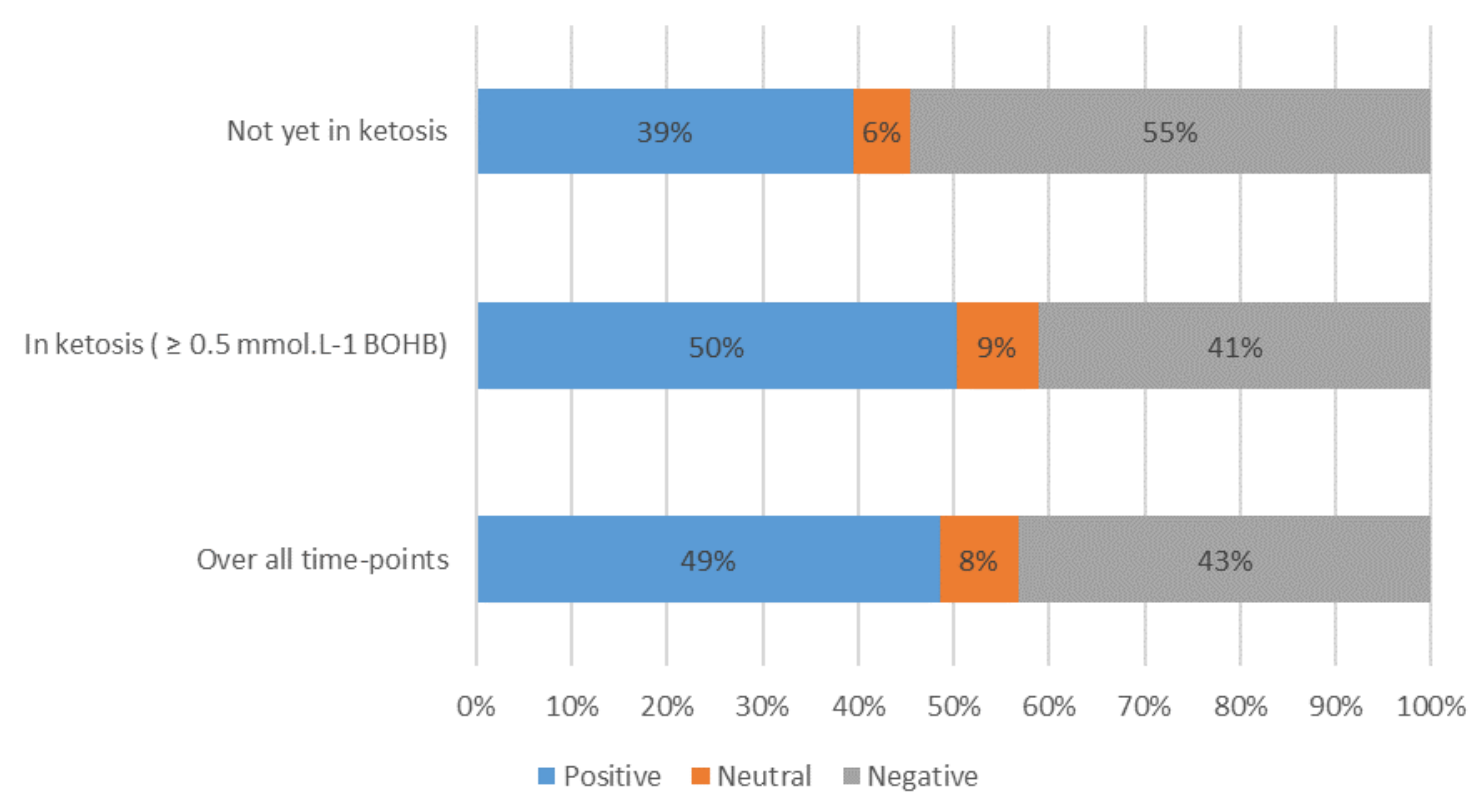

Figure 2. Percent of experiences classified as positive or negative during keto-induction, nutritional ketosis, and over the course of the study

\section{Physical effects}

Physical effects accounted for the largest category of reported responses. Gastrointestinal (GI) disturbances accounted for most adverse effects noted while on the diet. These included diarrhoea (the most commonly reported adverse effect), stomach cramps and constipation. Interestingly these GI effects were noted almost exclusively in the intervention group (those taking MCT oil). These symptoms, especially cramping and diarrhoea, are known effects associated with MCT supplementation. Ivy and colleagues have previously observed that $100 \%$ of participants in their study experienced gastric distress (cramping and diarrhoea) with dosages of 50 and $60 \mathrm{~g} \mathrm{MCT}$, with small effects at 30 g. $3^{2}$ Less frequently reported were headaches, muscular cramps and physical weakness and one participant experienced a nosebleed. One participant considered pulling out of the study (unbeknownst to the researchers and revealed in the post-study focus group) due to extreme symptoms; "I found it was very hard. Everything went wrong; headaches, shakes, dizzy, up all night just trying to get fluids in. "I honestly yes, almost quit because I felt it was dangerous." She sought medical advice and was provided with an electrolyte solution that caused the symptoms to abate. Interestingly, this participant stated that the 'hardship' of having these extreme symptoms and overcoming them, provided a reason to continue with the diet during, and after the study; "Probably because it ended [the physical symptoms], and after the ending, I had a recipe for success."

Some physical weakness was noted in association with exercise, but not concomitantly with lowered energy or mental cognition or mood. Instead, mood, energy, and cognition were improved (when compared to the perception of mood before the intervention), even though muscular weakness was present during exercise. Physical effects, in some cases, affected wellbeing; "Feeling low in 
energy, headachy, stomach pain after meals is not encouraging me to be overactive and isn't making [me] feel very happy.” Interestingly, one participant's asthma symptoms disappeared completely on the diet; "Asthma completely disappeared too."

\section{Mood, energy, and cognition}

Mood, energy, and cognition typically improved over the course of the diet. However, there were adverse effects on these at the beginning of the study. This mood and energy suppression is likely to be related to the lowered provision of fuel (especially glucose) during this period, and other factors (such as electrolyte disturbance). ${ }^{23-26}$ "I found that just at the start was the hardest part" "but then once I was [in ketosis] I was pretty fine."

Poor energy (fatigue and tiredness) were commonly reported in the early stages of the diet, when participants, for example, "felt lightheaded" "quite low in energy", "difficulty focusing and concentrating". Moreover, for some, the reduction in energy was profound; "Tired and wondering how on earth I'm going to do this for 20 days". However, for this respondent, and others, reported energy and mood did improve over the course of the study period. Conversely, some though suffered poor energy up until the end of the 20-day study; "bit lightheaded and tired, so everything seems to take a little more effort. Quite looking forward to finishing diet", and others made no mention of poor energy at all, instead, only stable or improved energy.

Changes in energy patterns were noted, with either reduced or improved energy in the morning; or a 'crash' in the evening. "For most of the day, I felt great, at the end of the day I really seemed to crash." Over the course of the study, energy mostly stabilised or improved for those reporting poor energy early in the study, along with improved or enhanced mental focus and clarity, e.g. "really noticing a steady energy level especially at night when I am not exhausted going to bed. I just know I need to sleep but not shattered and 'over it' which is how I used to feel" and "Feeling very even in my moods (as in no real highs or lows and more 'mindful')".

Irritability was noted by several participants, predominantly in the early days of the study, and characterised by comments such as "having a short fuse" and "tired with a short fuse". This irritability was also noted as a feeling of being 'wired,' i.e. "[I] felt bloated, wired and tired!" and was not necessarily related to increased energy, but instead, with fatigue. It is possible that this irritability and 'wired' feeling was related to increased sympathetic nervous system activity associated with carbohydrate withdrawal and the early keto-induction phase. This enhanced sympathetic nervous system activation, and increased epinephrine, norepinephrine, and cortisol has been observed in the early stages of carbohydrate-restricted diets and provides for the increased provision of glucose to preserve work capacity.33-35

Those who participated in exercise noted reduced energy in response to exercise, despite daily energy levels being stable or improved (in contrast to habitual peaks and troughs); "Got an hour's bike ride in and felt rooted from the start. Energy levels still stable but just low". For two participants this was also exacerbated by mistakenly not taking the prescribed amount of supplemental oil. Omitting or taking the incorrect dosage of the supplemental oils, albeit accidentally, is likely to have affected energy 


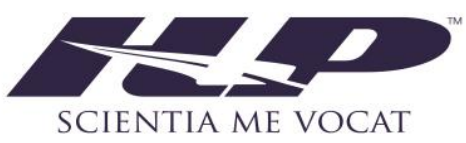

levels significantly, as the major provider of calories on a ketogenic diet are lipids.

Despite lower than normal perceived energy during-exercise earlier in the study, energy seemed to improve for participants over the duration of the study. For example, a participant noted on Day 3; "Hard CrossFit session but no energy," yet by Day 18 , despite being tired he did a "Hard CrossFit session" that he had "heaps of energy for!" Others shifted to a higher state of energy very quickly; "Feeling really good, energy levels are great!" (Day 4).

A potential confounding influence on energy levels was that calories were uniform based on a population- and age-appropriate eucaloric allocation of $2200 \mathrm{kcal}$ for males and $1800 \mathrm{kcal}$ for females. Thus, exercise would have provided a calorie deficit to hard-training participants. Training and exercise were not controlled in this free-living study.

It was also common for energy, mood and clarity to be improved even in the presence of negative physical effects such as diarrhoea, nausea and GI distress, and in the absence of quality sleep. "I must have got about 4 good night sleeps over the whole thing but the really weird thing was that during the day like as soon as I ate breakfast my energy levels came up and it was like I had a super power....”

Overall there seemed to be a general improvement in energy over the course of the study and a tendency towards improvements in 'stability' of energy following the VLCKD. While during-exercise energy did also improve over the course of the study, our impression was that this was reduced overall in relation to the diet.

\section{Satiety and hunger}

Satiety was drastically improved for most participants by the ketogenic diet. Ketogenic diets improve satiety, reduce hunger, and reduce the desire to eat. ${ }^{19}, 36$ Several participants were surprised at the volume of food, and especially vegetables, in the plan; "Food portions [are] actually more than I would normally eat so, pretty bloated after lunch" and "veggie portions are just too big". The vegetable portions recommended in the plan were approximately three cups with at least two meals per day to ensure that participants (who were not eating fruit due to the carbohydrate-restricted nature of the VLCKD) were consuming more than the recommended $5^{+}$per day vegetable and fruit recommendation, in accordance with dietary guidelines for health. The difficulty of consuming this amount speaks to a common difficulty in dietary planning in generalcompliance with vegetable and other 'nutrientdense' (fruit, berry) food intake recommendations. For example, the New Zealand Adult Nutrition Survey of 2009 showed that many New Zealanders fail to eat the recommended servings of vegetables and fruits, and fail to consume recommended amounts of several vitamins and minerals from diet alone. 37

This finding provides a hypothesis for vegetables as a 'crowding' food type, providing improved satiety and reducing the desire, within a meal, for other foods (such as optional 'fuel' foods-particularly sugars and starches). Interestingly, the absence of carbohydrate foods was perceived by some as a challenge not matched by how they felt. For instance, "I was concerned at the amount of food and being hungry, but I was pleasantly surprised that I felt full after each meal for many hours." 
Different portion sizes, in comparison to a habitual diet, appeared to be quite challenging. This was particularly evident in the morning when satiety was increased; "Finding it hard to eat so much at breakfast," "Feeling much less hungry, couldn't finish breakfast!" Sometimes this was accompanied by increased afternoon hunger; "extremely hungry after 3 pm" despite improved morning satiety. Feelings of fullness were sometimes associated with a lack of interest in food, characterised by comments such as "disinterested in food" and "no appetite."

The change in diet itself also caused some 'confusion' for participants in their perception of hunger. Several participants previously ate more and snacked more frequently. Thus, they wondered whether they did, in fact, need so much food or whether they were simply habituated to it. "It's difficult to tell if it's 'real' hunger (I don't think so) or just 'habit hunger." Because of this, some participants began to alter the diet - for example by consuming a smaller breakfast due to an inability to eat all the food prescribed.

A change was observed in response to snacking behaviours; "Normally I will be hungry for each meal especially when waking up in the morning or going longer than usual without a meal, and now I am finding this is not so much the case." For instance, a participant noted on the first day of the study that it was a "shock" not snacking, but the following day noted, "[I] am finding the not-snacking' easier than anticipated" and by the end of the study "Not so hungry before meals, not starving or constantly thinking about my next meal." Others noted similar surprise at the elimination of the need for snacking, e.g. "I was surprised at how well I coped given the lack of snacks I usually have" and the ability to go for much longer periods than used to without needing to eat meals or snack; "I'm used to eating every 3 hours" I went from $12.30 \mathrm{pm}$ to $6.30 \mathrm{pm}$ without eating-unheard of!!” Occasionally, hunger led to cravings and non-compliance with the diet, i.e. "Felt hungry at times and couldn't resist some bread and a few hot chips."

The supplemental oils prescribed for the study appeared to improve satiety and provide a sense of 'fullness.' So, in addition to accountability provided by being involved in a dietary study, the satiating effect of the oils was a factor contributing to compliance. "I think for me it wasn't that I was following a strict regime, it was just that the oil made me feel so full". The oils, however, were associated with physical symptoms of nausea and GI effects (as noted earlier).

Overall responses and effects of the diet were very positive; "I like that it's a good amount of calories and I'm not hungry", "I have felt full most of the time and have enjoyed eating more fat and protein", "I definitely don't feel hungry during the day on this diet".

In general, satiety was improved, with comments of 'fullness' common; "I feel full most of the time" and "I have to force myself to eat as not feeling hunger". Improved satiety positively affected cravings "I always feel full and no cravings", "I didn't crave the pizza or feel like I was missing out", "I feel so surprised at how full I've been feeling on this program."

\section{Cravings and temptation}

It has previously been demonstrated that lowcarbohydrate diets reduce cravings and desire for sugar and starches. $3^{8}$ We noted a general improvement in sugar cravings in the study. For 
example, a comment from Day 1; "craving sugar as I have a major sweet tooth" but by Day 7 the same participant noted; "Feeling great, no sugar cravings." Likewise, other comments indicated the reduced craving for sugar "Sugar/junk food cravings are seriously diminished. First time ever!" and "Not craving much at all" Not craving carbs, sugar", "not fantasising about custard squares!" Satiety, possibly enhanced by the supplemental oils, reduced sugar cravings; "Have noticed that sugar cravings come back with a vengeance if not having enough fat with each meal." Participants who reported a tendency to have sugar cravings (or the "sugar demon" as one participant put it) tended to agree that if you have a little, you cannot help but have more; "when you have carbs they make you crave more." "I feel so bad [when eating sugar], and the only thing that would make me feel better is to have more." Ingesting carbohydrate (especially sugar) improves mood in people who experience sugar cravings. 39 In these diets, carbohydrates are quite literally 'off the table' and cannot continue to drive the positive feedback loop of craving more sugar. Evidence links dopamine release in the mid-brain to the pathophysiology of psychosis, addiction and reward. Repeated ingestion of refined carbohydrate in a 'normal' western-style diet, stimulates the same dopaminergic pathway. 40

Other comments indicated a behavioural tendency towards a mixture of moderation and abstinence, e.g. "I would like to have one day where I can eat anything I like, but I am stricter on the other days like you know having that day to look forward to where I can eat anything." Others lamented the ability not to be able to moderate food intake; "I just wish I had the 'one bite ability,' you know, just to taste a cookie rather than a whole pack..."

Although we noted a strong, general improvement in reported sugar cravings, over the course of the study, we noted an increase in the desire for, but importantly, not craving for carbohydrates (starches). This pattern appeared to be related to a desire to return to more 'normal' dietary patterns.

Typically, during the study, cravings were resisted, when previously they may have been succumbed to; "Really want carbs ... bread and butter never looked so good! Have refrained", "desperately wanted to just eat rubbish. Pure psychological warfare. Funny. Mostly resisted"

Non-compliance with the diet was also associated with negative effects. "Eating off plan yesterday left me with a huge food hangover", and there was a reluctance to 'give in' to cravings, especially where there were high perceived rewards from the diet (especially weight loss). "Craving sugar today, but not wanting to go back to normal eating as I don't want to put the weight I've lost back on".

Cravings and temptation that did arise during the study did not appear to result from reduced satiety or increased hunger. "[I] was tempted with my son's porridge today but amazed that I'm stronger than my sugar cravings and they're going too."

Additionally, some participants became bored with the compendium of foods available; "Getting a bit bored with the food" and "Sick to death of meat and eggs."

Despite social situations providing a challenge, the diet, due to its restricted nature, enabled easier choices, especially at restaurants. "I felt 
like restaurants were fine as you can order fish or meat and then get two sides of vegetables". This suggests that nutrition education ('what' to eat) and enabling strong, self-determined decision, is critical to the nutrition counselling process, rather than a simple dietary prescription.

\section{Sleep}

Overall, references related to sleep indicated improved sleep quality while following the VLCKD. improvement; "Sleeping really well and feeling great." "Great, solid eight hours sleep." "I'm also falling asleep better, rather than tossing and turning how I used to." Improvements in sleep quality have been observed in children with epilepsy following a ketogenic diet, ${ }^{4}$ and ketogenic diets improve the GABA-glutamate ratio, providing a plausible explanation for greater relaxation and improved sleep..$^{2}$

Difficulty getting to sleep was occasionally noted by some, however. This was often in association with a 'racing heart' indicative of a high stress-response such as "I would wake up at about 2 am, so getting to sleep my muscles and everything felt quite restless, like jittery, and then I would get to sleep" or "Had difficulty getting to sleep and felt like I had a racing heart". As mentioned earlier, there are sympathetic nervous system effects during the early adaptation to a ketogenic diet, that might increase one's 'stress response'. One participant had extreme difficulties sleeping, noting early waking, trouble getting to sleep and sugar cravings; "finding that I am not sleeping well at all - waking often and too early...so I am getting tired only because I feel like I am not getting quality sleep", "really tired today - not sleeping well at all...huge sugar cravings - suspect due to tiredness...had to eat nuts to manage my way through". Interestingly, despite a real challenge with sleep, the participant felt good, and had improved mental clarity and focus; "really struggling with sleep...waking incredibly early and having trouble getting off to sleep at night. Otherwise feel good!", "tired yesterday from terrible sleep - still great mental clarity though," "Not sleeping well at all. Totally exhausted today. Even had a day sleep which is not like me at all. Still mental clarity though".

\section{Post-study reflections and intended changes.}

Participants in the post-study focus group all stated that they desired to continue on a reduced carbohydrate diet. Most commonly, they desired to continue to experience improved energy or cognition, reduced cravings and improved satiety, and weight loss. This was typified by statements such as; "I really enjoyed it. I lost weight; my skin cleared"...emotionally it was probably the most even-keeled I have felt in a really long time" and "I really liked the fact that I was not hungry, and I also wasn't emotional eating either, and I liked the kind of rigidity of the eating plan." Moreover, this appeared to cause a 'shift' in habits and a desire to educate oneself about lower-carbohydrate food options; "Because I felt so good, I felt like I need to carry this on, so I branched out and read a lot."

Our impression from the post-study focus group was that most participants were likely to continue to follow some variation of a carbohydrate-restricted nutrition plan. For example, "I have kind of stuck with it somewhat" I'm certainly doing a modified version", but without the heavy use of 
supplemental oil, choosing instead whole-food dietary fats and perhaps adding in some whole food, nutrient-dense carbohydrate types (such as kumara (sweet potato) and yams); "I found it hard that I couldn't have baked kumara so I was craving it when I would usually never crave that and I was kind of like 'give me the carbs!!' I feel long term a modified version of that plan would be really good."

Even when returning to a diet closer to habitual, higher-carbohydrate eating (albeit still lower in carbohydrate than previously consumed) there was a tendency to be mindful of carbohydrate portion size; "I'm still eating bread and rice but maybe not as much".

Greater attention to carbohydrate quantities in food could provide positive, long-term health benefits, as reduced carbohydrate diets are likely to be more effective for early weight loss for those obese and insulin resistant than comparable low-fat diets. ${ }^{43-46}$ Insulin resistant people also adhere more easily to lowcarbohydrate diets, and there is no detriment to adherence for those insulin sensitive. 47

Anecdotally, our clinical observations have indicated that cost can be perceived as a prohibitive factor to the use of ketogenic and lower-carbohydrate diets. Although this was not a stated outcome of our study, the relative costs of the diet vs habitual eating were mentioned in the post-study focus group. Those that didn't habitually eat meat and other highprotein, higher-fat, lower-carbohydrate foods found the diet more cost-prohibitive, e.g. "[Cost was] Maybe a bit more because I do not usually cook or buy meat much" and "I was eating more meat so maybe a little bit more costly". Whereas those that habitually ate more meat and processed and refined foods found it cheaper than their usual diet; "I thought it was cheap, especially as the protein/meat portions on a ketogenic diet are restricted." "And [[I'm] not eating as much meat as usual". A lower overall cost was also evident for those with a greater tendency to snack, compared to nonsnackers; "I feel like because I am [usually] eating so many snacks usually I found it actually quite cheap".

The consensus was that the diet intervention was a worthwhile experience for several reasons. Accountability was a major factor and is perhaps a confounding aspect of any dietary study; "if I wasn't in the study I would not have persevered". The accountability provided by the daily ketone readings had the effect of 'gamifying' the experience and people, therefore, wanted to comply. "You know with daily readings there was that sort of accountability with it". However, the ongoing cost (post-study) of the ketone testing strips provided a barrier to the continued use of this tool for accountability; "the sticks ran out just as I finished and so I went to the chemist to buy more and they are \$3.15 per stick and for me that was just way too much."

Being involved in the study and having 'choice' taken away was a powerful contributor to compliance; "What I have found is that the choice is actually taken away from you. Instead of thinking I could have one biscuit at home it is actually no gingernuts and you kind of think 'oh easy'. So, it isn't really a selfcontrol issue, because when it comes to sugar, I do not have any".

Self-care was also mentioned as a motivating factor; "another thing that was so great about doing this was that I was doing something for me, instead of doing something for everyone 
else in the household, I actually had the focus on me and what I was putting into my body and what I was doing for me and other people were sort of supporting me and they were supporting me with it and I really enjoyed that."

There was also a community aspect to this, both between the participants and within the extended communities of the participants. "People were always asking me what my numbers were, my brother or my mum or at work and you know everyone was quite interested you know how is it going kind of thing”.

\section{Discussion}

There were significant challenges faced by the participants in this study, particularly concerning physical effects. Many of these effects were GI and were mostly limited to the intervention group (taking MCT oil) for which GI effects are a known side effect. However, other side effects and physical symptoms also provided a significant challenge. Despite this, the most serious side-effects were experienced by people who subsequently became advocates for continuing the diet. This typically occurred once the keto-induction period ended, or methods had been found to mitigate the negative symptoms (such as additional electrolytes).

The overall perception of the diet was positive, and the 'lived experience' of the diet likewise provided benefits for the overall feeling of wellbeing and mood, along with improved sleep and reduced frequency of sugar and carbohydrate cravings.

Most participants in the focus group stated that they were continuing with some form of modified lower-carbohydrate diet due to the benefits, and this suggests that the lived experience' of a ketogenic diet overall is positive and that aspects of lower carbohydrate eating are both sustainable and may be preferable to habitual, higher-carbohydrate eating patterns.

Negative experiences diminished as participants adapted to a VLCKD, and so it is unlikely that a ketogenic diet per se causes negative effects. Rather, it is the adaptation to the diet and its differential fuel use, transient increased sympathetic nervous system activity, and most prominently electrolyte imbalance ${ }^{23-}$ ${ }^{26}$ that causes these challenges. These effects are minor and limited to the first 1-4 days of a ketogenic diet. ${ }^{23,}{ }^{27}$ More importantly, for some participants, the diet itself provided a near epiphany, in that it offered a dietary option that provided a greater degree of freedom from hunger and cravings, and offered satiety, and enhanced mood and wellbeing.

The results though were highly variable between individuals. Submissions also varied considerably in quantity and depth, and we cannot exclude the possibility that those more enamoured with the dietary approach were also more effusive in their daily diary entries. Likewise, the voluntary post-study focus group was attended by eight people out of the original 28 participants, and this is unlikely to provide a comprehensive view of the effects on the lived psychological, psychosocial and psychoemotional effects of a VLCKD. Despite this, the available qualitative data indicates that the experience of a ketogenic diet is a positive one.

There were several limitations to this study. Differences in compliance may have resulted from the free-living nature of this study. We did not adjust for exercise and activity, although 
participants were advised not to change their current exercise habits. Standardised diets (both for male and female) were provided per age- and gender-adjusted average requirements. Thus, those participants that were more active may have experienced the diet differently due to a relative calorie restriction. We also recognise the limitations of our convenience sample arising from the snowball method of recruitment, via our networks on a first-come basis, as this led to an almost entirely female cohort. There may be differences in the lived experience of a ketogenic diet between genders, but to our knowledge, this is not indicated in the literature, and while we do not have any reason to believe that the results would not apply similarly to men, our results should be interpreted conservatively.

We also recognise the limitations of qualitative research methods. While we sought to reduce bias by using an inductive, rather than a deductive approach to thematic identification, and through 'triangulation', checking and confirmation of codes by co-researchers, the research and analysis, by its very nature cannot be wholly objective, nor should it be.

The authors would like to humbly submit this work to the wider field of clinical nutrition for what it is, a qualitative appraisal by practitioner-researchers, of the experience of a ketogenic diet.

The lived experience of a ketogenic diet is likely to provide appreciable benefits for some people, but further research to better understand the individual tolerance and response to differing diets is warranted. In addition to this, further research to find methods to indicate clinically appropriate diets for individuals based upon physical, psychological or personality characteristics, either known or yet to be elucidated would provide a valuable tool for practitioners. 


\section{Abbreviations}

Very-Low Carbohydrate, Ketogenic Diet (VLCKD); Medium Chain Triglyceride (MCT); Long Chain Triglyceride (LCT); Nutritional Ketosis (NK); Low Carbohydrate, High Fat (LCHF); Diabetic Ketoacidosis (DKA); Profile of Mood States (POMS); Total Mood Disturbance Score (TMDS); Auckland University of Technology (AUT)

\section{Acknowledgements}

We are grateful to the participants in this study. We also acknowledge the support of our colleagues at the Human Potential Centre, AUT University.

\section{Author affiliations}

${ }^{1}$ Human Potential Centre, Auckland University of Technology, Auckland, NZ

\section{Conflict of interest}

The authors declare that they have no conflict of interest

\section{Authors' contribution}

$\mathrm{CJdCH}, \mathrm{GMS}$, and MW designed research; $\mathrm{CJdCH}$ conducted research; $\mathrm{CJdCH}, \mathrm{GMS}$, and $\mathrm{MW}$ analysed data; $\mathrm{CJdCH}$ wrote the paper, with editing assistance from GMS and MW. CJdCH had primary responsibility for final content. All authors read and approved the final manuscript.

\section{Funding statement}

All funding was provided by the Human Potential Centre, AUT.

Received: Accepted: Published:

\section{Copyright and licenses}

This work is licensed under the Creative Commons Attribution 4.0 International License. To view a copy of this license, visit

http://creativecommons.org/licenses/by/4.0/ or send a letter to Creative Commons, PO Box 1866, Mountain View, CA 94042, USA.

\section{References}

1. Lefevre F, Aronson N. Ketogenic diet for the treatment of refractory epilepsy in children: A systematic review of efficacy. Pediatrics. 2000;105(4):e46.

2. Keene DL. A systematic review of the use of the ketogenic diet in childhood epilepsy. Pediatr Neurol. 2006;35(1):1-5.

3. Levy RG, Cooper PN, Giri P, Pulman J. Ketogenic diet and other dietary treatments for epilepsy. The Cochrane Library. 2012.

4. Henderson CB, Filloux FM, Alder SC, Lyon JL, Caplin DA. Efficacy of the ketogenic diet as a treatment option for epilepsy: Meta-analysis. J Child Neurol. 2006;21(3):193-8.

5. Neal EG, Chaffe $H$, Schwartz RH, Lawson MS, Edwards N, Fitzsimmons G, et al. The ketogenic diet for the treatment of childhood epilepsy: a randomised controlled trial. Lancet Neurol. 2008;7(6):500-6.

6. Paoli A, Rubini A, Volek J, Grimaldi K. Beyond weight loss: a review of the therapeutic uses of very-lowcarbohydrate (ketogenic) diets. Eur J Clin Nutr. 2013;67(8):789-96.

7. Sumithran $P$, Proietto J. Ketogenic diets for weight loss: A review of their principles, safety and efficacy. Obes Res Clin Pract. 2008;2(1):1-13.

8. Maalouf M, Rho JM, Mattson MP. The neuroprotective properties of calorie restriction, the ketogenic diet, and ketone bodies. Brain research reviews. 2009;59(2):293-315.

9. Castro K, Faccioli LS, Baronio D, Gottfried C, Perry IS, dos Santos Riesgo R. Effect of a ketogenic diet on autism spectrum disorder: A systematic review. Research in Autism Spectrum Disorders. 2015;20:31-8.

10. Varshneya K, Carico C, Ortega A, Patil CG. The efficacy of ketogenic diet and associated hypoglycemia as an adjuvant therapy for high-grade gliomas: A review of the literature. Cureus. 2015;7(2):e251.

11. Kulak D, Polotsky AJ. Should the ketogenic diet be considered for enhancing fertility? Maturitas. 2013;74(1):10-3.

12. Bueno NB, de Melo ISV, de Oliveira SL, da Rocha Ataide T. Very-low-carbohydrate ketogenic diet v. low-fat diet for long-term weight loss: a meta-analysis of randomised controlled trials. $\mathrm{Br} J$ Nutr. 2013;110(07):1178-87.

13. Paoli A, Bosco G, Camporesi E, Mangar D. Ketosis, ketogenic diet and food intake control: a complex relationship. Frontiers in Psychology. 2015;6.

14. McClernon FJ, Yancy WS, Jr., Eberstein JA, Atkins RC, Westman EC. The effects of a lowcarbohydrate ketogenic diet and a low-fat diet on mood, hunger, and other self-reported symptoms. Obesity (Silver Spring, Md). 2007;15(1):182-7.

15. Johnstone AM, Horgan GW, Murison SD, Bremner DM, Lobley GE. Effects of a high-protein ketogenic diet on hunger, appetite, and weight loss in obese men feeding ad libitum. The American Journal of Clinical Nutrition. 2008;87(1):44-55.

16. Krebs HA. The regulation of the release of ketone bodies by the liver. Adv Enzyme Regul. 1966;4(0):339-53. 
17. Kitabchi AE, Umpierrez GE, Miles JM, Fisher JN. Hyperglycemic crises in adult patients with diabetes. Diabetes Care. 2009;32(7):1335-43.

18. Misra $S$, Oliver NS. Diabetic ketoacidosis in adults. BMJ. 2015;351.

19. Gibson A, Seimon R, Lee C, Ayre J, Franklin J, Markovic $T$, et al. Do ketogenic diets really suppress appetite? A systematic review and meta-analysis. Obes Rev. 2015;16(1):64-76.

20. Guerci B, Benichou $M$, Floriot $M$, Bohme $P$, Fougnot $S$, Franck $P$, et al. Accuracy of an electrochemical sensor for measuring capillary blood ketones by fingerstick samples during metabolic deterioration after continuous subcutaneous insulin infusion interruption in type 1 diabetic patients. Diabetes Care. 2003;26(4):113741.

21. Hartman AL, Vining EP. Clinical aspects of the ketogenic diet. Epilepsia. 2007;48(1):31-42.

22. Yancy WS, Jr., Olsen MK, Guyton JR, Bakst RP, Westman EC. A low-carbohydrate, ketogenic diet versus a low-fat diet to treat obesity and hyperlipidemia: A randomized, controlled trial. Ann Intern Med. 2004;140(10):769-77.

23. Hamwi GJ, Mitchell MC, Wieland RG, Kruger FA, Schachner SS. Sodium and potassium metabolism during starvation. Am J Clin Nutr. 1967;20(8):897-902.

24. DeFronzo RA, Goldberg M, AGus ZS. The effects of glucose and insulin on renal electrolyte transport. J Clin Invest. 1976;58(1):83.

25. DeFronzo RA. The effect of insulin on renal sodium metabolism. Diabetologia. 1981;21(3):165-71.

26. Tiwari S, Riazi S, Ecelbarger CA. Insulin's impact on renal sodium transport and blood pressure in health, obesity, and diabetes. American journal of physiology Renal physiology. 2007;293(4):F974-84.

27. Harber MP, Schenk S, Barkan AL, Horowitz JF. Alterations in carbohydrate metabolism in response to short-term dietary carbohydrate restriction. Am J Physiol Endocrinol Metab. 2005;289(2):E306-E12.

28. Kang HC, Chung DE, Kim DW, Kim HD. Early- and late-onset complications of the ketogenic diet for intractable epilepsy. Epilepsia. 2004;45(9):1116-23.

29. Chul Kang H, Joo Kim Y, Wook Kim D, Dong Kim H. Efficacy and safety of the ketogenic diet for intractable childhood epilepsy: Korean multicentric experience. Epilepsia. 2005;46(2):272-9.

30. National Science Foundation. How should research involving "snowball samples" be handled from a human subjects perspective? Arlington, Virginia, USA: National Science Foundation,; N.D [

31. Campbell B, Kreider RB, Ziegenfuss T, La Bounty $P$, Roberts $M$, Burke $D$, et al. International Society of Sports Nutrition position stand: protein and exercise. J Int Soc Sports Nutr. 2007;4:8-.

32. Ivy JL, Costill DL, Fink WJ, Maglischo E. Contribution of medium and long chain triglyceride intake to energy metabolism during prolonged exercise. Int J Sports Med. 1980;01(01):15-20.

33. Langfort J, Pilis W, Zarzeczny R, Nazar K, Kaciuba-Uściłko H. Effect of low-carbohydrate-ketogenic diet on metabolic and hormonal responses to graded exercise in men. J Physiol Pharmacol. 1996;47(2):361-71.
34. Fraser DD, Whiting S, Andrew RD, Macdonald EA, Musa-Veloso K, Cunnane SC. Elevated polyunsaturated fatty acids in blood serum obtained from children on the ketogenic diet. Neurology. 2003;60(6):1026-9.

35. Zajac A, Poprzecki S, Maszczyk A, Czuba M, Michalczyk M, Zydek G. The Effects of a Ketogenic Diet on Exercise Metabolism and Physical Performance in OffRoad Cyclists. Nutrients. 2014;6(7):2493.

36. McClernon FJ, Yancy WS, Eberstein JA, Atkins RC, Westman EC. The Effects of a Low-Carbohydrate Ketogenic Diet and a Low-Fat Diet on Mood, Hunger, and Other Self-Reported Symptoms. Obesity. 2007;15(1):182-

37. University of Otago and Ministry of Health. A Focus on Nutrition: Key findings of the 2008/09 New Zealand Adult Nutrition Survey. Wellington; 2011.

38. Martin CK, Rosenbaum D, Han H, Geiselman PJ, Wyatt HR, Hill JO, et al. Change in Food Cravings, Food Preferences, and Appetite During a Low-Carbohydrate and Low-Fat Diet. Obesity. 2011;19(10):1963-70.

39. Corsica JA, Spring BJ. Carbohydrate craving: A double-blind, placebo-controlled test of the selfmedication hypothesis. Eat Behav. 2008;9(4):447-54.

40. Thornley S, Russell B, Kydd R. Carbohydrate reward and psychosis: an explanation for neuroleptic induced weight gain and path to improved mental health? Curr Neuropharmacol. 2011;9(2):370-5.

41. Hallböök T, Lundgren J, Rosén I. Ketogenic Diet Improves Sleep Quality in Children with Therapy-resistant Epilepsy. Epilepsia. 2007;48(1):59-65.

42. Roy M, Beauvieux M-C, Naulin J, El Hamrani D, Gallis J-L, Cunnane SC, et al. Rapid adaptation of rat brain and liver metabolism to a ketogenic diet: an integrated study using (1)H- and (13)C-NMR spectroscopy. J Cereb Blood Flow Metab. 2015;35(7):1154-62.

43. Pittas AG, Das SK, Hajduk CL, Golden J, Saltzman E, Stark PC, et al. A low-glycemic load diet facilitates greater weight loss in overweight adults with high insulin secretion but not in overweight adults with low insulin secretion in the CALERIE Trial. Diabetes Care. 2005;28(12):2939-41.

44. Cornier MA, Donahoo WT, Pereira R, Gurevich I, Westergren R, Enerback $S$, et al. Insulin sensitivity determines the effectiveness of dietary macronutrient composition on weight loss in obese women. Obes Res. 2005;13(4):703-9.

45. Ebbeling CB, Leidig MM, Feldman HA, Lovesky $M M$, Ludwig DS. Effects of a low-glycemic load vs low-fat diet in obese young adults: a randomized trial. Jama. 2007:297(19):2092-102.

46. Gardner CD, Offringa LC, Hartle JC, Kapphahn K, Cherin R. Weight loss on low-fat vs. low-carbohydrate diets by insulin resistance status among overweight adults and adults with obesity: A randomized pilot trial. Obesity. 2016;24(1):79-86.

47. McClain AD, Otten JJ, Hekler EB, Gardner CD. Adherence to a low-fat vs. low-carbohydrate diet differs by insulin resistance status. Diabetes Obes Metab. 2013;15(1):87-90. 